\title{
Hue difference contours can be used in processing orientation information
}

\author{
ANN ELSNER \\ University of Oregon, Eugene, Oregon 97403
}

\begin{abstract}
In most studies of orientation processing, chromatic information and achromatic information have been combined or confounded. The present experiments investigated the relative sizes of tilt aftereffect induced by these two types of information. In these experiments, the tilt aftereffect is the error in adjusting a test contour to vertical, following the scanning of an inspection contour. For inspection and test contours identical except for orientation, the tilt aftereffects varied with inspection contour orientation but not with chromatic or achromatic condition. Smaller tilt aftereffects were obtained when the inspection contour was produced by a hue difference (chromatic information) and the test contour was produced by a luminance difference (achromatic information), or vice versa. These results indicate that achromatic and chromatic information is processed in a similar manner with respect to orientation. Furthermore, there is substantial, but incomplete, pooling of chromatic and achromatic orientation information.
\end{abstract}

A contour at the border between two hues (hue difference contour) may appear less distinct than a contour at the border between two different luminances (luminance difference contour) (Boynton, 1973; Koffka \& Harrower, 1931a, 1931b; Liebmann, 1927). Analogously, chromatic information alone is insufficient for resolving very high spatial frequency information (DeValois \& DeValois, 1975; Granger \& Heurtley, 1973; Hilz \& Cavonius, 1970; van der Horst, de Weert, \& Bouman, 1967), while achromatic information is sufficient (Campbell \& Robson, 1968). These two types of findings, along with the assumption that high spatial frequency information is necessary in pattern recognition, may have led to the current trend of combining or confounding chromatic and achromatic information in form perception experiments.

To separate the effects of chromatic and achromatic information in orientation processing, the present experiments investigated the effect of hue difference or luminance difference test and inspection contours on the size of the tilt aftereffect. The tilt aftereffect is the error in the perceived orientation of a test contour, following the scanning of an inspection contour. This paradigm was chosen because the stimuli used are suprathreshold, McCollough effect conditioning procedures (McCollough, 1965) are unnecessary, and the results from various methods are similar or predictable

This research was supported by PHS Biomedical Sciences Grant 5 S07 RR07080-12 and was performed as part of the doctoral requirements at the University of Oregon. The author wishes to express her appreciation to Dr. Jacob Beck, Frances Tally, and Dr. Terry Hines for their assistance and suggestions. The author's present address is: Eye Research Laboratories, The University of Chicago, 950 East 59th Street, Chicago, Illinois 60637.
(Campbell \& Maffei, 1971; Gibson, 1937; Gibson \& Radner, 1937; Lovegrove \& Over, 1973; Mitchell \& Muir, 1976; Vernon, 1934). Reviews are given by Coltheart (1971) and Over (1970). Reliably, the test contour appears tilted an extra $1^{\circ}$ to $4^{\circ}$ away from the inspection contour (direct effect) when the contours are separated by $10^{\circ}$ to $20^{\circ}$ (maximum at $12.5^{\circ}$ ). Less reliably, the test contour appears tilted an extra $0.5^{\circ}$ to $1^{\circ}$ toward the inspection contour (indirect effect) when the contours are separated by $70^{\circ}$ or $80^{\circ}$.

Of particular relevance are these findings with luminance difference gratings: (1) The tilt aftereffect is smaller if the inspection and test gratings differ in color than if they are the same (Broerse, Over, \& Lovegrove, 1975; Lovegrove \& Over, 1973). (2) The tilt aftereffect is smaller if the test and inspection gratings differ in spatial frequency than if they are the same (Ware \& Mitchell, 1974). (3) If the inspection grating is low contrast and the test high contrast, the tilt aftereffect is smaller than if the two gratings are the same contrast, which, in turn, is smaller than if the inspection is high contrast and the test low contrast (Parker, 1972; Tolhurst \& Thompson, 1975). (4) The tilt aftereffect is invariant over color, contrast, and spatial frequency when test and inspection stimuli are the same (Lovegrove \& Over, 1973; Parker, 1972; Ware \& Mitchell, 1974).

\section{GENERAL METHOD}

\section{Observers}

The observers, eight University of Oregon students, had normal color vision by the H-R-R or Ishihara pseudoisochromatic plates. The seven paid observers had good acuity and minimal astigmatism. The author was corrected to normal. 


\section{Apparatus and Stimuli}

Each inspection and test stimulus was a circular bipartite field, $2.5^{\circ}$ in visual angle. The hemifields differed in hue or luminance. The border between the hemifields served as the inspection or test contour. Since the whole bipartite field could be rotated about its center, this border provided the orientation information of the inspection or test contour. Black paper or Kodak Wratten filters red 26 , green 58 , yellow 73 , blue 47 , and neutral density 96 determined the hue of each hemifield. A $45-\mathrm{W}$ quartz halide lamp and diffuser (correlated color temperature, $2,976^{\circ} \mathrm{K}$ ) transilluminated the bipartite field. The bipartite field appeared in a black surround, $138 \mathrm{~cm}$ away. A gray cone surrounded the observer's head and occluded all but the central $7^{\circ}$ of the visual field. A shutter obscured the stimulus change.

Luminance matching for the chromatic stimuli was done by four methods. Four observers performed brightness matches. A skilled observer made luminance estimates with an SEI photometer and measurements with a Pritchard spectraphotometer. These methods differed by not more than $.1 \log$ unit, except for the blue stimuli. ${ }^{1}$ Furthermore, the output of the source as measured by a Gamma Scanning Spectraradiometer was multiplied by the filter transmissions. Each product was multiplied by the relative spectral luminous efficiency for normal observers (Judd \& Wyszecki, 1975). The luminance matches fell between the calculations from a broad efficiency curve and those from a narrow one.

Contrast for the achromatic conditions was computed by $C=\left(L_{\max }-L_{\min }\right) /\left(L_{\max }+L_{\min }\right)$.

\section{Procedure}

A verbal ready signal preceded each trial. During the 1-min inspection, the observer horizontally scanned the contour to lessen afterimages. Following rapid stimulus change, the observer adjusted the test contour from a random starting position to perceived vertical, then relaxed.

Four practice adjustments preceded each block of 9 to 12 trials. The test contour was the same for a block. For each repetition of a stimulus condition, inspection contours were selected randomly without replacement, except that clockwise inspection orientations alternated with counterclockwise ones. Sessions lasted about $1 \mathrm{~h}$, with one per day.

\section{EXPERIMENT 1}

The purpose of Experiment 1 was to induce tilt aftereffects with hue difference contours and compare these aftereffects to effects induced by luminance difference contours.

\section{Method}

Observers. Four observers participated four times in each condition.

Apparatus and Stimuli. Three chromatic and two achromatic conditions were tested. In each condition, the inspection and test stimulus were the same except for contour orientation. The luminances given were measured by the Pritchard Spectraphotometer. In the red-green condition, the red hemifield was on the observer's right and the green on the left, with luminances of 890 and $690 \mathrm{~cd} / \mathrm{m}^{2}$. The green-red condition was identical, except that the green was on the right. Thus, these two conditions provide a measure of baseline variability. The red-1-green condition was identical to the red-green condition except for the luminance match, which was $690 \mathrm{~cd} / \mathrm{m}^{2}$ for both red and green. In the gray condition, the right hemifield was a darker gray than the left, 390 and $1,580 \mathrm{~cd} / \mathrm{m}^{2}$, respectively, with a contrast of .60 . In the black-white condition, the right hemifield was black, the left luminous white, and luminances were .69 and $3,800 \mathrm{~cd} / \mathrm{m}^{2}$, respectively, with a contrast of 1,0 .

The inspection contour orientations were $80^{\circ}, 70^{\circ}, 60^{\circ}, 50^{\circ}$, $40^{\circ}, 30^{\circ}, 20^{\circ}, 15^{\circ}, 10^{\circ}$, and $5^{\circ}$ clockwise and counterclockwise from vertical, plus vertical. Following the $1-\min$ inspection period, there was $1 \mathrm{~min}$ for the observer to make his adjustment (usually in less than $5 \mathrm{sec}$ ) and unadapt.

\section{Results}

The data were transformed by subtracting the setting following inspection of a vertical contour from each setting to remove experimental and perceptual artifacts. The resulting tilt aftereffect is plotted as a function of inspection orientation in Figure 1. Each point represents the mean of the four observer's median data. For both chromatic and achromatic conditions, these functions are similar to the achromatic ones in the published literature, except that counterclockwise inspection stimuli may produce larger tilt aftereffects than clockwise ones. This constant error is somewhat less than $.5^{\circ}$ and may be artifactual. The average function for each condition represents the individual data well. The sample standard deviations for each observer are similar to those across observers, generally about $.2^{\circ}$ to $.6^{\circ}$.

Ten separate analyses of variance were performed, one for each pair of inspection orientations. The factors were observer, condition, and direction (clockwise vs. counterclockwise). ${ }^{2}$ Significant tilt aftereffects were found with $5^{\circ}, 10^{\circ}, 15^{\circ}, 20^{\circ}$, and $30^{\circ}$ inspection orientations, and with $\mathrm{F}(1,3)$ ps were less than $.025, .01, .005, .05$, and .01 , respectively. That is, the test contour was adjusted to the inspection contour side of perceived vertical. The largest effect was for $15^{\circ}$ inspection orientations, a $2.2^{\circ}$ difference between counterclockwise and clockwise settings. This effect did not differ significantly from the $10^{\circ}$ inspection orientation effect. Indirect effects were found with $70^{\circ}$ and $80^{\circ}$ inspection orientations, $\mathrm{p}$ less than .001 and .005 , respectively. That is, the test contour was adjusted slightly farther away from the inspection contour than perceived vertical, with $.61^{\circ}$ and $.67^{\circ}$ differences between clockwise and counterclockwise inspection orientations.

The tilt aftereffect function for each condition was so similar to the others that there was only one Direction by Condition interaction that approached significance. With $30^{\circ}$ inspection contours, blackwhite produced the largest tilt aftereffect and redgreen the next $[F(4,12)=2.76, p<.1]$. Since a different ordering was found for $20^{\circ}$ and $40^{\circ}$ inspection contours, the interaction probably resulted from chance. Thus, the tilt aftereffect is invariant across the chromatic and achromatic conditions in this experiment.

All stimuli produced strong contours (sharp borders) when initially viewed. Mach bands, which enhanced the border of the achromatic stimuli, were often seen, mainly with the black-white condition. Similar effects were seen with all three chromatic conditions, but usually with hue or saturation changes at the border. These transitory effects did not enhance 


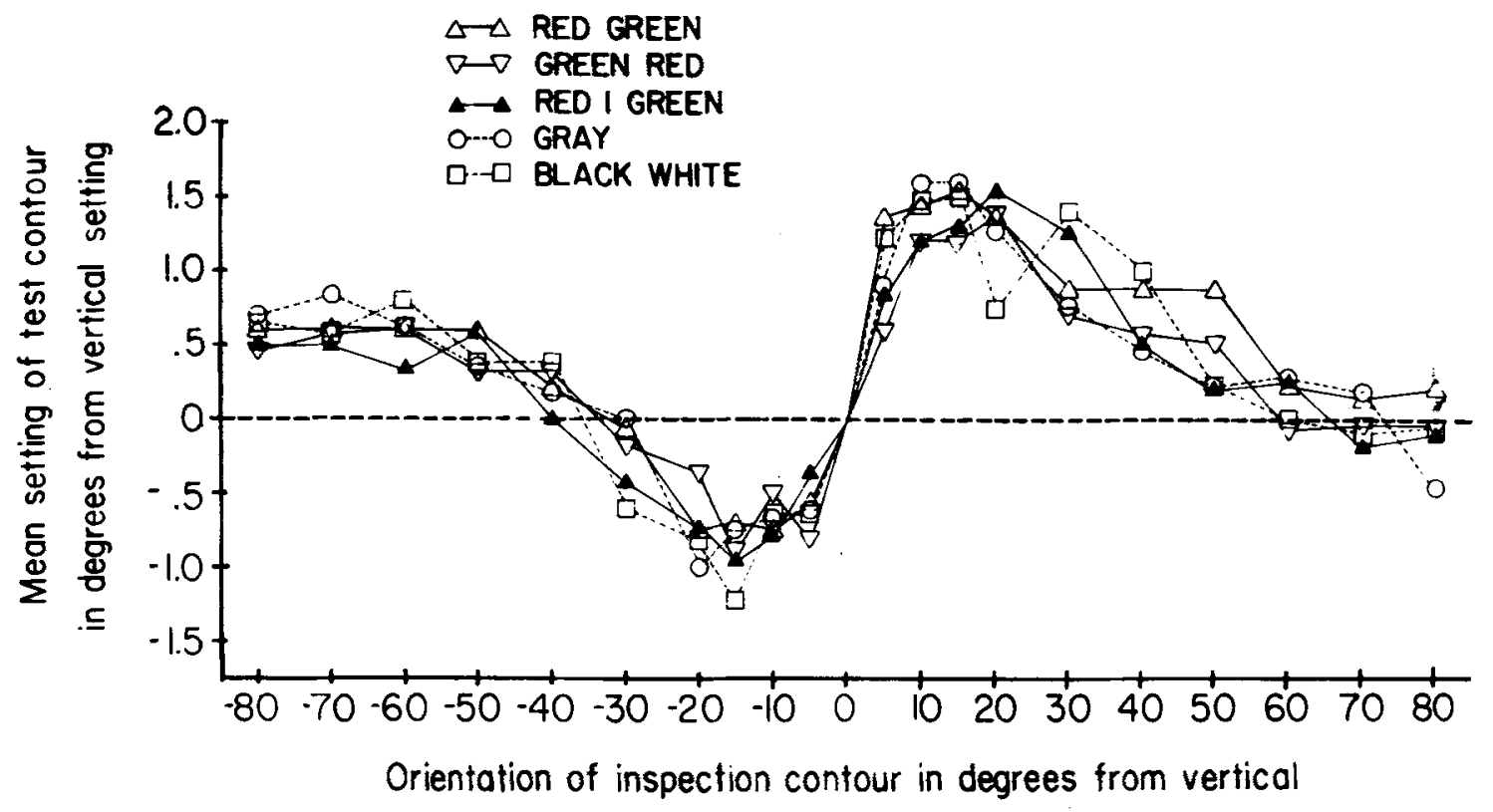

Figure 1. The mean difference from vertical setting in degrees is plotted as a function of inspection orientation. $A$ " + " indicates counterclockwise orientations with respect to the observer, a "-" clockwise ones. The conditions are represented as follows: $\triangle \longrightarrow \Delta$ red-green, $\nabla \longrightarrow \nabla$ green-red, $\sim \sim$ red-1-green, $\bigcirc \longrightarrow$ gray, and $\square \longrightarrow$ black-white.

the border and could have been due to chromatic adaptation, eye movements, or lateral inhibition. While chromatic aberration may have been present, it did not enhance the border with a bright stripe. The chromatic stimuli appeared somewhat desaturated and yellowed at the end of the inspection. No afterimages were seen while the test contour was in view.

\section{EXPERIMENT 2}

Additional measurements of the invariance of the tilt aftereffect across stimuli were made. Three color combinations and one achromatic stimulus were tested. According to previous studies (Comerford, 1974; Koffka \& Harrower, 1931a, 1931b), the stimuli selected should have contours of different strengths, blue-yellow being weak, red-green stronger, and black-white the strongest.

\section{Method}

Observers. Six observers participated five times in each condition.

Apparatus and Stimuli. One achromatic and three chromatic conditions were tested. In the red-green condition, the red hemifield was $34 \mathrm{~cd} / \mathrm{m}^{2}$ and the green $41 \mathrm{~cd} / \mathrm{m}^{2}$. In the yellow-blue condition, the yellow hemifield was $34 \mathrm{~cd} / \mathrm{m}^{2}$ and the blue $31 \mathrm{~cd} / \mathrm{m}^{2}$. In the red-blue condition, the red hemifield was $34 \mathrm{~cd} / \mathrm{m}^{2}$ and the blue $31 \mathrm{~cd} / \mathrm{m}^{2}$. In the black-gray condition, the black hemifield was $0.10 \mathrm{~cd} / \mathrm{m}^{2}$ and the gray $22 \mathrm{~cd} / \mathrm{m}^{2}$, contrast 1.0 .

The three inspection contour orientations were $10^{\circ}$ clockwise and counterclockwise, plus vertical. Two minutes between inspection periods were allowed for setting the test and the disappearance of afterimages.
Procedure. To determine contour strength, two ranking tasks were employed. One task was to rank the distinctness of the contour, a 1 indicating the most distinct contour (the most encoding of high spatial frequency information). A second task was to rank the apparent difference of the bipartite field halves, ignoring the border. A 1 indicated the most difference, and may be comparable to the highest contrast stimulus among a group of low spatial frequency stimuli.

\section{Results}

The setting following inspection of a vertical contour was subtracted from each adjustment for each repetition of a condition. The average of the medians of each observer is shown in Table 1 for each condition, the means representing the individual data well. A three-way analysis of variance, Observers by Direction (clockwise vs. counterclockwise) by Condition indicated significant tilt aftereffects $[F(1,5)=$ $66.25, p<.001]$. The average difference of adjustments following clockwise and counterclockwise inspection contours was $2.5^{\circ}$. There was no Condition by Inspection Orientation interaction. This indicates that the tilt aftereffects did not differ across conditions.

The mean ranks are also shown in Table 1. The rank order for neither distinctness of contour nor the difference between halves predicts the invariance of the tilt aftereffects. A three-factor Friedman analysis on the ranks, Condition by Ranking Task by Observer gave a significant main effect of condition $\left[\chi^{2}(3)\right.$ $=8.37, \mathrm{p}<.05]$. There was a significant Condition by Ranking Task interaction $\left[\chi^{2}=18.08, p<.001\right]$. indicating a different ordering for the two tasks. 
Table 1

Results from All Three Tasks of Experiment 2

\begin{tabular}{clcccc}
\hline & & & & \multicolumn{2}{c}{ Condition } \\
\cline { 4 - 6 } Task & & Black Gray & Red Green & Red Blue & Yellow Blue \\
\hline 1 & Distinctness of Contour & 1.0 & 2.8 & 3.0 & 3.2 \\
2 & Difference Between Halves & 3.2 & 2.2 & 1.2 & 3.5 \\
3 & Tilt Aftereffect & 2.53 & 2.28 & 2.59 & 2.53 \\
\hline
\end{tabular}

Note-(1) Average rank of the distinctness of the contour between bipartite field halves, a "1" indicates the most distinct contour. (2) Average rank of the apparent difference between bipartite field halves, a " 1 " indicates the most difference between halves. (3) Difference of the mean tilt aftereffects following counterclockwise vs. clockwise inspection contours, in degrees.

Hence, a separate Friedman analysis was performed on the data from each task. In the distinctness of contours ranking task, the expected order (most to least), black-gray $>$ red-green $>$ red-blue $>$ yellowblue, was significant $\left[\chi^{2}(3)=11.0, p<.01\right]$. In the difference-between-halves task, the order of red-blue $>$ red-green $>$ black-gray $>$ yellow-blue was significant $\left[\chi^{2}(3)=12.0, \mathrm{p}<.01\right]$. Thus, neither average ranking order predicts the invariance of the average tilt aftereffects. Linear contrasts performed on the individual data from each ranking task along with the tilt aftereffect data also indicate no relationship between contour strength and tilt aftereffect.

As before, anomalous color and saturation bands were present. The blue semicircle sometimes smeared over its borders.

\section{EXPERIMENT 3}

The invariance of the tilt aftereffect has been demonstrated for a variety of stimuli, provided the inspection and test are the same. Tilt aftereffects are obtainable when the inspection and test stimuli differ, but these are generally smaller. In Experiment 3, tilt aftereffects were sought with an achromatic test contour and a chromatic inspection contour, or vice versa. These tilt aftereffects could then be compared with the baseline conditions in which the inspection and test were both chromatic or both achromatic.

Another interpretation for these data is suggested by the tilt aftereffects induced when the test and inspection contour differ in contrast (Parker, 1972; Tolhurst \& Thompson, 1975). If an inspection grating is high contrast and the test low contrast, the tilt aftereffects are larger than if the inspection and test are the same contrast. In addition, if the inspection grating is low contrast and the test high contrast, the tilt aftereffects are minimal. Now assume that the chromatic tilt aftereffects are due solely to small luminance differences. Then inspecting a highcontrast contour and testing with a red-green (lowcontrast) one should produce large tilt aftereffects. Medium tilt aftereffects should be obtained when the inspection and test contours are both high con- trast or both red and green. Minimal tilt aftereffects should be obtained after inspecting the red and green contour and testing with the high-contrast one.

\section{Method}

Observers. Six observers participated four times in each condition.

Apparatus and Stimuli. One chromatic, one achromatic, and two mixed (chromatic and achromatic) conditions were tested. The stimuli were the red-1-green-condition and the gray-condition stimuli of Experiment 1 . In the red-green/red-green condition, the observer inspected and tested with the chromatic stimulus. In the gray/gray condition, the observer inspected and tested with the achromatic stimulus. In the red-green/gray condition, the observer inspected the chromatic stimulus but tested with the gray one. In the gray/red-green condition, the observer inspected the achromatic stimulus but tested with the chromatic one.

Five inspection orientations were tested, $10^{\circ}$ and $15^{\circ}$ clockwise and counterclockwise from vertical, plus vertical. The time between inspection periods was $1 \mathrm{~min}$, as in Experiment 1.

\section{Results}

The setting after inspecting a vertical contour of the same condition was subtracted from each setting. The data shown in Table 2 are difference scores for each condition, the mean settings following clockwise inspection orientations subtracted from the mean settings following counterclockwise ones. A five-way analysis of variance, Observers by Inspection Condition by Test Condition by Direction (clockwise vs. counterclockwise) by Distance from vertical $\left(10^{\circ} \mathrm{vs}\right.$. $15^{\circ}$ ), indicated highly significant tilt aftereffects $[F(1,5)=64.4, p<.001]$. That is, the settings

Table 2

Difference Between Mean Setting Following Counterclockwise Inspection Orientations and Clockwise Ones, in Degrees from Vertical Setting

\begin{tabular}{|c|c|c|c|c|}
\hline \multirow{2}{*}{ Type of Test } & \multicolumn{4}{|c|}{ Type of Inspection } \\
\hline & \multicolumn{2}{|c|}{ Red Green } & \multicolumn{2}{|r|}{ Gray } \\
\hline $\begin{array}{l}\text { Red Green } \\
\text { Gray }\end{array}$ & $\begin{array}{l}2.3 \\
1.8\end{array}$ & $\begin{array}{l}\text { (Medium) } \\
\text { (Small) }\end{array}$ & $\begin{array}{l}2.1 \\
2.4\end{array}$ & $\begin{array}{l}\text { (Large) } \\
\text { (Medium) }\end{array}$ \\
\hline
\end{tabular}

Note-The mean is the average of each observer's median score for that condition. The size of the tilt aftereffect if the red green stimuli were effectively low contrast stimuli is given in parenthesis. Means of 10 and $15 \mathrm{deg}$. 
following clockwise inspection contours were significantly different from those following counterclockwise ones.

The Inspection Condition by Test Condition by Inspection Orientation interaction was significant $[F(1,5)=17.0, p<.01]$. This indicates that the tilt aftereffects were different sizes in different conditions. The pattern was gray/gray $\simeq$ red-green/ red-green $>$ gray/red-green $>$ red-green/gray (see Table 2). Recall that the pattern predicted, if the red-green tilt aftereffects were due solely to a luminance difference, was gray/red-green $>$ gray/gray $\simeq$ red-green/red-green $>$ red-green/gray. This first pattern, besides being statistically significant, more or less describes each observer's data, except for one observer at only one distance from vertical. This interaction (and pattern of results) was replicated in a fourth study, even though the stimuli were half the luminance, the achromatic stimulus contrast was 1.0 , and the inspection period was only $5 \mathrm{sec}^{3}$

A three-way interaction, Distance from Vertical by Inspection Condition by Test Condition $[F(1,5)=$ $7.55, \mathrm{p}<.05]$ may be due to variability in the redgreen/gray and gray/red-green data across observers.

The mean tilt aftereffects obtained in Experiments 1 and 3 were compared for four observers who participated in both experiments. For all equivalent conditions, there was no significant difference between means or standard deviations.

Both stimuli in this experiment produced strong contours. Afterimages were not visible during the adjustment of the test contour.

\section{DISCUSSION}

Tilt aftereffects were obtained with contours having maximal chromatic and minimal achromatic information. What is the role of the chromatic information in inducing tilt aftereffects? Consider Experiment 1. Assuming that residual luminance information was responsible for the chromatic tilt aftereffects implies that stimuli matched for luminance should produce little or no tilt aftereffect. If the redgreen condition were not a good luminance match for a given observer, the red-1-green condition (.1 log unit dimmer) might have been. Thus, for that observer, the red-1-green tilt aftereffects should have been much smaller than the red-green ones. However, there was no difference between red-green and red-1green tilt aftereffects for any observer. Thus, residual luminance information was probably not solely responsible for the chromatic tilt aftereffects in Experiment 1.

Next consider Experiment 2. A low luminance contrast may have existed across the chromatic contours. Yet, with the light-adapted conditions, the low stimulus luminance, and only one contour present to detect, the luminance contrast alone would have been near or below threshold. Furthermore, the achromatic contour always was ranked more distinct than the chromatic ones, indicating much greater luminance contrast in the achromatic stimulus. Thus, it is doubtful that luminance information alone was responsible for the chromatic tilt aftereffects.

The analysis of Experiment 3 supports this notion. If the achromatic stimulus were effectively a high luminance contrast and the chromatic stimulus a low luminance contrast, the achromatic inspection stimulus should have produced larger tilt aftereffects with the chromatic test stimulus than the achromatic one. Hence, the gray/red-green condition tilt aftereffects should be larger than the gray/gray ones. This was not the case. Generally, the gray/gray-condition tilt aftereffects were larger than the gray/red-green ones. Again, residual luminance information was not solely responsible for the tilt aftereffects in chromatic or mixed conditions.

A better explanation of the data is that orientation processing is done with both chromatic and achromatic information. These two types of information are combined, but with some loss. The amount of loss varies across observers. Hence, the tilt aftereffects are smaller when the inspection and test contours differ, i.e., the red-green/gray condition and the gray/red-green condition. This finding for chromatic vs. achromatic information is similar to the findings that smaller tilt aftereffects are found when inspection and test stimuli differ in color (Broerse, Over \& Lovegrove, 1975; Lovegrove \& Over, 1973) or spatial frequency (Ware \& Mitchell, 1974) than when they do not.

If the inspection and test stimuli do not differ, the tilt aftereffect is invariant over color, contrast, or spatial frequency (Lovegrove \& Over, 1973; Parker, 1972; Ware \& Mitchell, 1974). The results of all three experiments indicate that this finding holds for chromatic vs. achromatic stimuli as well. Note the similarity for the chromatic vs. achromatic tilt aftereffect functions at all inspection orientations in Figure 1, Experiment 1 . If these tilt aftereffect functions can be considered as measures of orientation tuning, then under these experimental conditions the orientation tuning is as sharp for chromatic as for achromatic stimuli.

The results of Experiment 2 show invariance of the tilt aftereffect over three chromatic and one achromatic conditions. The modulation transfer functions of achromatic, red and green, and yellow and blue stimuli (Campbell \& Robson, 1968; Granger \& Heurtley, 1973; Hilz \& Cavonius, 1970; van der Horst, de Weert, \& Bouman, 1967) imply that the contours should differ in distinctness. The predicted order of black and gray more distinct than red and green, red and green more distinct than yellow and blue, 
was found. Yet the tilt aftereffect did not differ across stimuli. This finding for contour distinctness agrees with the invariance of the tilt aftereffect over a range of spatial frequencies (Ware \& Mitchell, 1974) and that tilt aftereffects are obtained following inspection of subjective contours (Smith \& Over, $1975,1976)$. Note that all contours in the study were fairly distinct. The invariance of the tilt aftereffect might fail with indistinct contours, highly desaturated stimuli matched for luminance, or two stimuli of nearly equal wavelengths. That is, unless a strong enough difference signal of chromatic or achromatic information exists, a tilt aftereffect may not occur.

The tilt aftereffect was also invariant across luminance. The stimuli in Experiment 2 were about $1 / 20$ the luminance of those in Experiments 1 and 3. Comparable tilt aftereffects were obtained over this luminance range.

\section{SUMMARY}

Chromatic information alone or with only minimal luminance information is used in orientation processing in a manner similar to achromatic information. The two types of information are combined, but with some loss.

\section{REFERENCES}

BoYNTon, R. Implications of the minimally distinct border. Journal of the Optical Society of America, 1973, 63, 1037-1043.

Broerse, J., Over, R., \& LOVEGROVE, W. Loss of wavelength selectivity in contour masking and aftereffect following dichoptic adaptation. Perception \& Psychophysics, 1975, 17, 333-336.

Camprell, F. W., \& Maffei, L. The tilt aftereffect: A fresh look. Vision Research, 1971, 11, 833-840.

Campbell, F. W., \& Robson, J. G. Application of Fourier analysis to visibility of gratings. Journal of Physiology, 1968, 197, 551-556.

Coltheart, M. Visual feature-analyzers and aftereffects of tilt and curvature. Psychological Review, 1971, 78, 114-121.

COMERford, J. Stereopsis with chromatic contours. Vision Research, 1974, 14, 974-982.

DeVAlors, R. L., \& DEVAlois, K. K. Neural coding of color. In E. C. Carterette \& M. P. Friedman (Eds.), Handbook of Perception, V. Academic Press: New York, 1975.

Gibson, J. J. Adaptation, after-effect and contrast in the perception of tilted lines. II. Simultaneous contrast and the areal restriction of the after-effect. Journal of Experimental Psychology, 1937, 20, 553-569.

GibSON, J. J., \& RADNER, M. Adaptation, after-effect and contrast in the perception of tilted lines. I. Quantitative studies. Journal of Experimental Psychology, 1937, 20, 453-467.

Granger, E. M., \& Heurtley, J. C. Visual chromaticitymodulation transfer function. Journal of the Optical Society of America, 1973, 63, 1173-1174.

Hulz, R., \& Cavonius, C. R. Wavelength discrimination measured with square wave gratings. Journal of the Optical Society of America, 1970, 60, 273-277.
Jund, D. B., \& Wyszeck, G. Color in business, science and industry. Wiley: New York, 1975.

KofFKA, K., \& HARRower, M. R. Colour and organization I. Psychologische Forschung, 1931, 15, 145-192. (a)

KoffKa, K., \& Harrower, M. R. Colour and organization II. Psychologische Forschung, 1931, 15, 193-275. (b)

Liemmans, S. Uber das Verhalten farbiger Formen bei Helligkeitsgleichheit von Figure und Grund. Psychologische Forschung, 1927, 9, 300-353.

Lovegrove, W. N., \& Over, R. Colour selectivity in orientation masking and aftereffect. Vision Research, 1973, 13, 895-903.

McCollougr, C. Color adaptation of edge detectors in the human visual system. Science, $1965,179,1115-1116$.

Mitchell, D. E., \& Muir, D. W. Does the tilt aftereffect occur in the oblique meridian? Vision Research, 1976, 16, 609-613.

OVER, R. Comparison of normalization theory and neural enhancement explanation of negative aftereffects. Psychological Bulletin, $1970,75,225-243$.

Parker, D. M. Contrast and size variables in the tilt aftereffect. Quarterly Journal of Experimental Psychology, 1972, 24, 1-7.

SMITH, A. T., \& OvER, R. Tilt aftereffects with subjective contours. Nature, 1975, 257, 581-582.

SMITH, A. T., \& OvER, R. Color-selective tilt aftereffects with subjective contours. Perception \& Psychophysics, 1976, 20. 305-308.

TolmuRst, D. J., \& Thompson, P. G. Orientation illusions and aftereffects. Inhibition between channels. Vision Research, 1975, 15, 967-972.

Van der Horst, G. J., de Weert, C. M. M., \& Bouman, M. A. Transfer of spatial chromaticity-contrast at threshold in the human eye. Journal of the Optical Society of America, 1967, 57, 1260-1266.

Vernon, M. D. The perception of inclined lines. British Journal of Psychology, 1934, 25, 186-196.

W ARE, C., \& Mitchell, D. E. The spatial selectivity of the tilt aftereffect. Vision Research, 1974, 735-736.

\section{NOTES}

1. The blue stimuli appeared .1 to $.2 \log$ unit brighter than the yellow stimuli at the luminance match. When the brightness difference was reduced, the tilt aftereffect results were comparable on the one observer tested.

2. The $\mathrm{O}$ by $\mathrm{IC}$ by $\mathrm{C}$ by $\mathrm{D}$ factorial experiment was considered as a set of $10 \mathrm{O}$ by $\mathrm{C}$ by $\mathrm{D}$ factorial experiments. Planned comparisons allow insufficient degrees of freedom to independently test simple main effects of direction and simple interactions for each inspection orientation. Although testing. simple main direction effects by testing the main effect of a reduced portion of the data is a conservative procedure, significant results were obtained where predicted from published literature. Because the Condition by Direction interaction $F$ ratios were similarly small across inspection orientations, little would be changed by a more complex measure for the interaction $F$ ratio.

3. Similar size tilt aftereffects were obtained for same or different inspection and test contours using (1) the red-green and greenred stimuli of Experiment 1 and (2) the red-green, yellow-blue, and gray stimuli of Experiment 2. Quantitative data analysis was impossible due to the confusing afterimages seen during the adjustment.

(Received for publication December 15, 1977; revision accepted August 28, 1978.) 